Notfall Rettungsmed 2018 $\cdot 21: 664-672$ https://doi.org/10.1007/s10049-018-0516-6 Online publiziert: 17. September 2018 (c) Der/die Autor(en) 2018

\section{Redaktion}

M. Bernhard, Düsseldorf

H. Helm, Ulm

T. Wurmb, Würzburg

CrossMark

T. Wurmb $\cdot$ B. Kowalzik ${ }^{2}$ J. Rebuck ${ }^{4}$ A. Franke ${ }^{3}$ D. Cwojdzinski ${ }^{5} \cdot$ N. Bernstein ${ }^{6}$. T. Brodala $\cdot$ M. Weber ${ }^{7}$

${ }^{1}$ Sektion Notfall- und Katstrophenmedizin, Klinik und Poliklinik für Anästhesiologie, Universitätsklinikum Würzburg, Würzburg, Deutschland

${ }^{2}$ Referat Schutz der Gesundheit, Bundesamt für Bevölkerungsschutz und Katastrophenhilfe, Bonn, Deutschland

${ }^{3}$ Klinik für Orthopädie und Unfallchirurgie, Wiederherstellungs- und Handchirurgie und Verbrennungsmedizin, Bundeswehrzentralkrankenhaus Koblenz, Koblenz, Deutschland

${ }^{4}$ Gesundheitswirtschaft, Hochschule für angewandte Wissenschaften Kempten, Kempten, Deutschland

${ }^{5}$ Senatsverwaltung für Gesundheit, Pflege und Gleichstellung, Berlin, Deutschland

${ }^{6}$ Fachbereich Bundespolizei, Hochschule des Bundes für öffentliche Verwaltung, Lübeck, Deutschland

${ }^{7}$ Akademie für Krisenmanagement, Notfallplanung und Zivilschutz, Bundesamt für Bevölkerungsschutz und Katastrophenhilfe, Bad Neuenahr-Ahrweiler, Deutschland

\title{
Bewältigung von besonderen Bedrohungslagen
}

\section{Ergebnisse des bundesweiten Auswerteprozesses am Bundesamt für Bevölkerungsschutz und Katastrophenhilfe - Teil 1: Präklinisches Management}

richtigen Lehren daraus zu ziehen. Die Analyse der bisherigen Anschläge und die Definition der zu ziehenden Konsequenzen wurden in enger Zusammenarbeit mit benannten Vertretern der an der Bewältigung von lebensbedrohlichen Einsatzlagen beteiligten Organisationen und Institutionen formuliert.

\section{Definition gemeinsamer Ziele} anschläge und ein folgenschwerer Amoklauf verübt.

Im Auftrag des Bundesministeriums des Inneren, für Bau und Heimat und des Bundesministeriums für Gesundheit übernahm das Bundesamt für Bevölkerungsschutz und Katastrophenhilfe (BBK) die Planung und Durchführung eines strukturierten Auswerteprozesses, um die jüngsten Anschläge aus dem eigenen Land und aus den europäischen Nachbarländern zu analysieren und die

Für Rückfragen bezüglich des Auswerteprozesses steht Ihnen Dr. Martin Weber (martin.weber@bbk.bund.de) zur Verfügung.
Das gemeinsame Formulieren von Zielen hat für die Einsatzvorbereitung und den konkreten Einsatzablauf eine wesentliche Bedeutung. Nur so können die Einsatzkräfte die Einsatzschwerpunkte der jeweils anderen Organisationen verstehen und ihr eigenes Handeln danach ausrichten. Dies hilft im Einsatz die Kommunikation auf einen wesentlichen Informationsaustausch zu reduzieren, konfliktträchtige Naht- und Schnittstellen im Vorfeld zu identifizieren sowie diese zu entschärfen; darüber hinaus hilft es, das gegenseitige Verständnis zu stärken und das Vertrauen zu fördern. Ein gemein- sames Ziel zu verfolgen, ist der Schlüssel zum Erfolg [1-3].

Die größte der besonderen Herausforderungen der jüngsten Anschläge in Europa ergibt sich aus der Tatsache, dass es sich zumeist um eine fortgesetzte Attacke durch Täter handelt, die bereit sind, im Rahmen ihrer Aktion zu sterben. Alle beteiligten Organisationen müssen zur selben Zeit im selben Raum unter einer konkreten und mehrdimensionalen Bedrohung agieren, um die folgenden definierten Ziele zu erreichen:

- Erhalt und Wiederherstellung der gesellschaftlichen Integrität,

- Rettung möglichst vieler Menschen und

- größtmöglicher Schutz der Einsatzkräfte.

\section{Festlegung einer Strategie zur Erreichung der Ziele}

Um das definierte Ziel zu erreichen, muss eine Strategie gewählt werden, die auf die besonderen Umstände einer lebens- 
bedrohlichen Einsatzlage ausgerichtet ist.

Hierbei spielen die

- fortgesetzte Bedrohung,

- die Möglichkeit eines zweiten Schlages (sog. „second hit"),

- die Verwendung von Kriegswaffen und die

- die durch diese Waffen verursachten Verletzungen

eine entscheidende Rolle [4].

Wie schon in anderen Auswerteprozessen beschrieben [5] wurde für das präklinische Management lebensbedrohlicher Einsatzlagen folgende Strategie festgelegt:

- beende oder kontrolliere die Bedrohung,

- finde die roten Patienten und

- „clear up the scene immediately“.

Beende die Bedrohung. Eine wesentliche Grundlage für die Rettung möglichst vieler Menschen ist die Einwirkung auf den oder die Täter zur Beendigung der Bedrohung [1-3]. Diese wird ausschließlich von Polizeikräften ausgeführt.

\section{》) Wesentlich für die erfolgreiche Einsatzbewältigung ist die Festlegung gemeinsamer Ziele und Strategien}

Sehr wohl können aber die Kräfte der nichtpolizeilichen Gefahrenabwehr - gerade in Fällen, in denen sie vor der polizeilichen Gefahrenabwehr am Ereignisort eintreffen und/oder die Lage (noch) nicht als Terroranschlag/Amoklauf eingestuft wurde - selbst in den Fokus des/der Täter geraten. Daher kommt der gemeinsamen Lageeinschätzung, der schnellen und zielgerichteten Kommunikation dieser Einschätzung und dem Eigenschutz der Einsatzkräfte eine besondere Bedeutung $\mathrm{zu}$.

\section{Finde die lebensbedrohlich verletzten} Patienten (Sichtungskategorie I Rot).

Um möglichst viele Opfer zu retten, ist die schnelle Identifikation der lebensbedrohlich verletzten Patienten essenziell.

Diese Patienten müssen im Rahmen einer Vorsichtung der Sichtungskatego- rie SK I Rot zugeordnet werden. Da die Art der Gewaltanwendung im Rahmen von Terroranschlägen zumeist penetrierende Verletzungen verursacht, wird es maßgeblich Patienten mit kritischen Blutungen geben, die schnellstmöglich zur chirurgischen Therapie in Krankenhäuser gebracht werden müssen [5-10]. Gleiches gilt letztlich aber auch für schwere stumpfe Verletzungen, wie man sie nach einem Überrolltrauma findet.

„Clear up the scene immediately“. Diese Strategie hat zwei wesentliche Aspekte: Zum einen wird der Imperativ erfüllt, lebensbedrohlich blutende Patienten schnellstmöglich in ein Krankenhaus zu bringen, zum anderen ist der beste Eigenschutz für Einsatzkräfte und Schutz der Betroffenen die kürzest mögliche Verweildauer am Einsatzort [5].

Die hier vorgestellte Arbeit stellt die präklinische Patientenversorgung als einen Teilaspekt des gesamten Auswerteprozesses dar. Die anderen Teilaspekte werden in eigenen Veröffentlichungen dargestellt.

\section{Methodisches Vorgehen}

Zum Thema Bewältigung lebensbedrohlicher Einsatzlagen gab es unter anderem im Jahre 2016 zunächst zwei Veranstaltungen mit BBK-Beteiligung: das 5. Forum Interdisziplinäre Zusammenarbeit im Gesundheitlichen Bevölkerungsschutz an der Akademie für Krisenmanagement, Notfallplanung und Zivilschutz (AKNZ) im Mai 2016, initiiert vom Bundesamt für Bevölkerungsschutz und Katastrophenhilfe (BBK), und die von Frankreich initiierte „Lessons-learned-Veranstaltung“ zu den Anschlägen in Paris an der französischen Botschaft in Berlin, zusammen mit dem Bundesministerium des Inneren für Bau und Heimat (BMI) und dem Bundesministerium für Gesundheit (BMG) in Kooperation mit dem BBK im September 2016.

Im Nachgang zu diesen beiden Veranstaltungen wurde am BBK eine Projektgruppe eingesetzt, um diesen besonderen Herausforderungen auf nationaler Ebene begegnen zu können. Das BBK als Prozessleitung und -steuerung fungier-
Hier steht eine Anzeige. 글 Springer 
Notfall Rettungsmed 2018·21:664-672 https://doi.org/10.1007/s10049-018-0516-6

(c) Der/die Autor(en) 2018

T. Wurmb · B. Kowalzik · J. Rebuck · A. Franke · D. Cwojdzinski · N. Bernstein · T. Brodala · M. Weber

Bewältigung von besonderen Bedrohungslagen. Ergebnisse des bundesweiten Auswerteprozesses am Bundesamt für Bevölkerungsschutz und Katastrophenhilfe - Teil 1: Präklinisches Management

\section{Zusammenfassung}

Hintergrund und Methoden. Terroristische

Anschläge und Amoktaten stellen die Einsatzkräfte der polizeilichen- und nichtpolizeilichen Gefahrenabwehr vor große Herausforderungen. Mit dem Ziel, die gelernten Lektionen aus den bisherigen Einsätzen in Deutschland zu definieren und bestehende Konzepte an neue Formen der Bedrohungen anzupassen, wurde am Bundesamt für Bevölkerungsschutz und Katastrophenhilfe (BBK) ein mehrstufiger Auswerteprozess durchgeführt. Am BBK wurden mehrere Ergebniskonferenzen zu unterschiedlichen Themenkomplexen durchgeführt und die Ergebnisse jeweils schriftlich formuliert. Beteiligt waren Vertreter der relevanten Akteure zur Bewältigung solcher Lagen wie Behörden, Institutionen, Organisationen sowie nationale Experten. Die hier vorgestellten Ergebnisse beziehen sich auf den Themenkomplex der präklinischen Patientenversorgung.

Ergebnisse. Die Vorbereitung auf lebensbedrohliche Einsatzlagen kann nur als schnittstellen- und organisationsübergreifendes Konzept gelingen. Ein vorhandenes Konzept für den Massenanfall von Verletzten (MANV) oder Erkrankten stellt eine essenzielle Basis für die erfolgreiche Bewältigung einer lebensbedrohlichen Einsatzlage dar. Die Spezifika des taktischen und medizinischen Vorgehens, die sich durch die Besonderheiten einer lebensbedrohlichen Einsatzlage ergeben, müssen auf den MANV-Konzepten aufbauen und werden hier detailliert vorgestellt.

Schlussfolgerung. Die präsentierten Konsequenzen sind das Resultat eines strukturierten Auswerteprozesses auf nationaler Ebene. Sie stammen von Experten der an der Bewältigung lebensbedrohlicher Einsatzlagen beteiligten Institutionen und Organisationen. Damit stellen sie eine Expertenmeinung dar, die auf der Basis aller bisher vorliegenden Erkenntnisse gebildet wurde.

Schlüsselwörter

Terroranschlag · Massenanfall von Verletzten . Amok · Evaluation · Rettungskräfte

\section{Management of mass killing incidents. Results of a nationwide evaluation by the German Federal Office of Civil Protection and Disaster Assistance-Part 1: prehospital management}

\begin{abstract}
Background and methods. Terrorist attacks and rampages are extremely challenging situations for police and rescue forces. With the aim to define the lessons learned from the latest terrorist attacks in Germany and neighbouring European countries, the German Federal Office of Civil Protection and Disaster Assistance conducted a nationwide multilevel evaluation on behalf of the Federal Ministry of the Interior and the Federal Ministry of Health. In close collaboration with all organisations and institutions directly involved in the management of terrorist attacks or rampages, a number of conferences with thematic
\end{abstract}

priorities were held in order to formulate conclusions and recommendations. The results of the conference "prehospital casualty care" are presented in this publication. Results. Essential for the successful management of life-threatening mass casualty incidents is a well-trained concept for conventional mass casualty incidents. Specific conditions of rescue missions for terrorist attacks or rampages must be identified and the tactic consequences should complete the existing concepts. The specific conditions and the resulting consequences are described in the result section of this publication.
Conclusion. The presented lessons learned are the results of a nationwide evaluation process conducted by the Federal Office of Civil Protection and Disaster Assistance. It is seen as an expert opinion and might be of interest for all organizations and institutions dealing with the management of terrorist attacks or other life-threatening rescue missions.

\section{Keywords}

Terrorism · Mass casualty incidents · Rampage . Lessons learned $\cdot$ Emergency responders te hierbei als Plattform für einen interdisziplinären Austausch aller beteiligten Institutionen, Organisationen und Verbände.

Der gesamte Prozess wurde in $4 \mathrm{Ab}$ schnitte gegliedert:

1. Auftaktveranstaltung: Der Bedarf eines strukturierten Auswerteprozesses wurde festgelegt und die thematischen Cluster gebildet, die im weiteren Verlauf bearbeitet werden sollten.

2. Identifikation von Fähigkeitslücken in Bezug auf die Herausforderungen lebensbedrohlicher Einsatzlagen:
Festlegung der Themenfelder und der zu bearbeitenden Fragestellungen.

3. Durchführung von 4 Ergebniskonferenzen an der AKNZ zu den Themen:

a. Erkennen und Alarmieren,

b. Kommunikation und taktisches Verhalten,

c. klinische Patientenversorgung, d. präklinische Patientenversorgung.

4. AKNZ-Fachkongress:

a. Vorstellung der Ergebnisse,

b. Entwicklung von konkreten Werkzeugen zur Umsetzung der Ergebnisse, c. Einbeziehung vorhandener Konzepte zur psychosozialen Notfallversorgung (PSNV) und psychosoziales Krisenmanagement,

d. Erarbeitung der Themenfelder Ausbildung und Ressourcen unter Einbeziehung der Teilnehmer des Fachkongresses.

Die Einbeziehung von CBRN-Lagen (Chemisch, Biologisch, Radiologisch oder Nukleär) in die Erarbeitung von Ergebnissen ist grundlegender Bestandteil des Prozesses. Auf eine umfassende Bearbeitung des CBRN-Themenkomple- 
xes musste aufgrund der vorgegebenen zeitlichen Begrenzung im Rahmen der Ergebniskonferenzen verzichtet werden. Dieses Thema wird zu einem späteren Zeitpunkt entsprechend bearbeitet.

In dem hier vorgestellten Manuskript werden die Ergebnisse des 3. Prozessschritts zum Thema Präklinische Patientenversorgung dargestellt. Basierend auf den Vorarbeiten der vorangegangenen Arbeitsschritte (wie oben bereits beschrieben) hat sich die Ergebniskonferenz auf die Erarbeitung von Lösungsmöglichkeiten für folgende Bereiche der präklinischen Versorgung konzentriert:

1. MANV-Konzepte für besondere Bedrohungslagen,

2. Behörden und Organisationen mit Sicherheitsaufgaben (BOS-)übergreifende Konzepte,

3. Raumordnung,

4. Ersteinschätzung, Vorsichtung und Sichtung,

5. Rolle der Polizei,

6. medizinische Versorgungsstrategien,

7. Transport (v. a. bei penetrierenden Traumata),

8. besondere Patientengruppen,

9. Umgang mit unverletzten Betroffenen,

10. Erfassung von Patientendaten.

\section{Ergebnisse des Auswerte- prozesses}

\section{MANV-Konzepte für besondere Bedrohungslagen}

Die aus den Ergebnissen der Konferenz abgeleiteten Empfehlungen richten sich grundsätzlich an alle Vertreter der polizeilichen und nichtpolizeilichen Gefahrenabwehr und sollten angepasst an die jeweils regionalen und lokalen Regelungen Strukturen umgesetzt werden.

Ein gut ausgearbeitetes, geschultes und trainiertes Konzept für den Massenanfall von Verletzten (MANV) oder Erkrankten (MANI) stellt eine essenzielle Basis für die erfolgreiche Bewältigung einer lebensbedrohlichen Einsatzlage dar. Die Spezifika des Vorgehens, die sich durch die Besonderheiten einer lebensbedrohlichen Einsatzlage ergeben, müssen auf den allgemeinen bestehen-

den MANV-Konzepten aufbauen und diese ergänzen.

Die Erarbeitung von MANV-Konzepten liegt im Verantwortungsbereich der kommunalen Ebene/Landesebene unter Beteiligung aller Behörden und Organisationen mit Sicherheitsaufgaben (BOS).

\section{\) Ein gut ausgearbeitetes MANV-Konzept ist Basis für die Bewältigung lebensbedrohlicher Einsatzlagen}

Es wird betont, dass der Katastrophenschutz maßgeblich vom Ehrenamt getragen wird. Eine Einbindung und Förderung des Ehrenamts ist unabdingbar, um die Basis des Katastrophenschutzes und damit auch die Fähigkeit zur Bewältigung von Großschadensereignissen und lebensbedrohlichen Einsatzlagen langfristig zu sichern. Dies gilt für die Erstellung von Konzepten gleichermaßen wie für die Schulungen und Übungen.

\section{BOS-übergreifende Konzepte}

Die Vorbereitung auf lebensbedrohliche Einsatzlagen kann nur als schnittstellen- und organisationsübergreifende Konzeption gelingen. Die Festlegung gemeinsamer Ziele und gemeinsamer Strategien ist eine essenzielle Voraussetzung für die erfolgreiche, gemeinsame Bewältigung lebensbedrohlicher Einsatzlagen. Die Ziele und die Strategien zum Erreichen dieser Ziele wurden oben bereits beschrieben.

Wesentliche Punkte für das gemeinsame Vorgehen von polizeilicher und nichtpolizeilicher Gefahrenabwehr sind folgende:

- Ein gemeinsames Lagebild ist die essenzielle Grundlage für taktische Entscheidungen und wesentlicher Bestandteil einer gemeinsamen Lagebewältigung. Hierzu ist ein schneller und direkter Informationsaustausch zwischen den beteiligten Kräften vor Ort sowie rückwärtig unverzichtbar.

- Informationsaustausch zwischen den Leitstellen von Polizei und Feuerwehr/Rettungsdienst zum frühestmöglichen Zeitpunkt. Hieraus resultiert die Entwicklung eines abgestimmten Vorgehens im Einsatz (z. B. abgestimmte Anfahrt der Kräfte, abgestimmte Festlegung von Bereitstellungsräumen/Kräftesammelstellen).

- Einrichtung, Sicherstellung und Verwendung eines prioritären Kommunikationskanals zwischen den jeweiligen Leitstellen, z. B. rotes Telefon mit definiertem Sender und Empfänger (Informationsbündelung an definierten Stellen).

- Kommunikation der Einsatzführungskräfte vor Ort zu einem frühestmöglichen Zeitpunkt.

- Austausch von Verbindungspersonen in den Stäben zu einem frühestmöglichen Zeitpunkt.

- Gewährleistung der Erkennbarkeit der Führungskräfte vor Ort durch eindeutige Kennzeichnung.

- Gemeinsames Festlegen einer Raumordnung durch Polizei, Rettungsdienst und Feuerwehr. Hierbei Anwendung des Prinzips „10 für 10“. Dies bedeutet, dass die Investition einer kurzen Analysezeit (symbolisch $10 \mathrm{~s}$ ) mit dem Resultat einer abgestimmten und abgewogenen Entscheidung sich im weiteren Verlauf zeitlich deutlich auszahlt (symbolisch $10 \mathrm{~min}$ ).

- Festlegung des unmittelbaren Gefahrenbereichs aufgrund der vorliegenden Informationen und Lagebewertung durch die Polizei. In diesem unsicheren Bereich agiert nur die Polizei.

- Bevorratung von Tragehilfen und Materialien zur Blutstillung in sofort verfügbaren Einsatzfahrzeugen. Diese werden frühestmöglich an den Schadensort verlegt.

- Gemeinsame Festlegung von Materialübergabepunkten, z. B. Tragehilfen (Bergetücher, Spineboards etc.) zur Rettung von Patienten und Betroffenen aus dem unsicheren Bereich (Start der Transportkette, s. unten).

- Start der Transportkette aus dem unsicheren Bereich in Richtung sicherer Bereich. Festlegen von Übergabepunkten (von Polizei an Rettungsdienst) für Verletzte und unverletzt Betroffene. 
Ersteinschätzung - Vorsichtung und Sichtung - Raumordnung Rolle der Polizei

In der Initialphase einer lebensbedrohlichen Einsatzlage sind zwei Informationen wesentlich: Wie ist die Gefährdungslage und mit wie viel Betroffenen ist $\mathrm{zu}$ rechnen? Hierzu sind eine erste gemeinsame strukturierte Gefährdungsbeurteilung durch ersteintreffende Einsatzkräfte und die Kommunikation des Ergebnisses dieser Einschätzung an die jeweiligen Leitstellen - und zwischen den Leitstellen - sowie nachfolgende Einsatzkräfte essenziell. Aus dieser Gefährdungsbeurteilung wird die Raumordnung abgeleitet und der unsicherere Bereich festgelegt, in dem nur die Polizei agiert.

\section{》) In der Anfangsphase des Einsatzes muss die Polizei auf den oder die Täter einwirken}

In der Anfangsphase des Einsatzes ist es die Hauptaufgabe der Polizei, auf den oder die Täter einzuwirken, um die Gefahr zu kontrollieren und einen Übergang in eine mobile Lage $\mathrm{zu}$ unterbinden.

Für die Ressourcenplanung des Rettungsdienstes ist die Kenntnis der ungefähren Anzahl schwer/lebensbedrohlich verletzter Opfer wichtig. Diese Information kann in der Initialphase in der Regel nur die Polizei erheben und weitergeben. Das Ziel dieser Ersteinschätzung ist der Erhalt einer Grundlage für weitere taktische Entscheidungen (z.B. Anfordern von Transportkapazität bei einer hohen Anzahl lebensbedrohlich verletzter $\mathrm{Pa}$ tienten). Sichtung und Vorsichtung finden nicht durch die Polizei und nicht im Gefahrenbereich statt. Bei der Ersteinschätzung findet keine Kennzeichnung der Opfer/Betroffenen statt; als lebensrettende Sofortmaßnahme sollte aber das Stillen kritischer Blutungen durchgeführt werden.

Außerdem muss in der frühen Phase eine gerichtete Transportkette in Richtung der sichereren Bereiche in Gang gesetzt werden. Die Polizei unterstützt durch entsprechende verbale Aufforderungen unverletzte/leicht verletzte $\mathrm{Be}$ troffene dabei, sich selbst gerichtet aus dem Gefahrenbereich in Richtung sicherer Bereiche zu entfernen.

\section{Ersteinschätzung und Start der Transportkette}

- Abschätzen der Anzahl der Betroffenen durch Polizei, Rettungsdienst oder Feuerwehr in Abhängigkeit von den Gefahrenbereichen (• Abb. 2)

- Abschätzung der Anzahl schwer verletzter Patienten

- Unverzügliche Meldung des Ergebnisses der Ersteinschätzung an die jeweilige Leitstelle

- Start der gerichteten Transportkette im unsicheren Bereich durch die Polizei

Eine Vorsichtung findet frühestens im teilsicheren oder sicheren Bereich durch medizinisches Personal statt. Die Vorsichtung wird algorithmenbasiert durchgeführt. Eine Festlegung auf einen speziellen Algorithmus und eine entsprechend Empfehlung kann aufgrund der Datenlage nicht gegeben werden [11]. Hierzu wird außerdem auf die Ergebnisse der 7. Sichtungs-Konsensus-Konferenz verwiesen [12]. Im verwendeten Vorsichtungsalgorithmus sollte in jedem Falle ein Warnmechanismus mit Hinweis auf eine eventuelle Gefahrenlage und den daraus resultierenden Eigenschutz enthalten sein.

\section{Die Ziele und Konsequenzen der Vorsichtung}

- Schnellstmögliche Identifikation und Retten der lebensbedrohlich verletzten Patienten (SK I rot)

- Entscheidung zur weiteren Ordnung des Raums (z. B. Einrichtung weiterer Patientenablagen, Rettungsmittelhalteplatz, Ladezone)

- Durchführung lebensrettender Sofortmaßnahmen:

- Stillen von Blutungen

- Freihalten der Atemwege

- Entlastung thorakaler Spannungssituationen

- Lagerung

- Gut sichtbare Kennzeichnung aller vorgesichteten Patienten

- Fortführen der gerichteten Transportkette
- Schnellstmöglicher Abtransport der Patienten mit der Sichtungskategorie SKI Rot

\section{Medizinische Versorgungs- strategien}

Das Ausmaß der medizinischen Versorgung wird durch die aktuelle Lage bestimmt und nicht durch das Ausmaß der Verletzungen. Dies entspricht den Grundsätzen der taktischen Medizin. Um diesbezüglich angemessene Entscheidungen treffen zu können, ist das Vorliegen eines aktuellen Lagebilds von größter Bedeutung. Davon ausgehend wird die Raumordnung festgelegt und ein unsicherer, ein teilsicherer und ein sicherer Bereich unterschieden.

Unsicherer Bereich: Hier können nach aktuellem Konsens erste medizinische Maßnahmen nur durch die Polizei durchgeführt werden. Der Schwerpunkt liegt auf der Blutstillung durch einfache Maßnahmen.

Teilsicherer Bereich: In diesem Bereich findet die Übergabe der Patienten an den Rettungsdienst statt. Hier können geschützte Patientenablagen eingerichtet werden (s. Diskussion). In diesem Übergabebereich finden die Vorsichtung und weitere lageabhängige medizinische Versorgungen statt (erweiterte lebensrettende Sofortmaßnahmen, z.B. erweitertes Atemwegsmanagement oder Entlastung eines Spannungspneumothorax).

Sicherer Bereich (evtl. erst im Krankenhaus): Hier findet ressourcenabhängig die weiterführende Patientenversorgung statt. Im sicheren Bereich wird die Sichtung durchgeführt. Es bestand Einigkeit darüber, dass taktische Einheiten, wie z. B. ein Behandlungsplatz am Schadensort nicht eingesetzt werden sollten. Dies ist mit der Strategie „clear up the scene immediately" nicht vereinbar.

Bei der Planung und Durchführung der medizinischen Versorgung müssen die Besonderheiten bei den zu erwartenden Verletzungsmustern beachtet werden. Bei einem Einsatz von Kriegswaffen und Sprengmitteln ist mit einem überproportional hohen Anteil von lebensbedrohlich blutenden Patienten zu rechnen. Dies bedeutet, dass initial blutstillende Maßnahmen (Druckverband, 
Tourniquet) bei peripheren Blutungsquellen lebensrettend sind. Bei zentralen Blutungsquellen (Hals, Thorax, Abdomen, Leiste, Achselhöhle) muss ein schnellstmöglicher Transport in das nächste geeignete Krankenhaus erfolgen, um dort eine chirurgische Blutstillung zu erzielen.

\section{Typische Verletzungen bei terroristischen Anschlägen}

1. Blutung durch penetrierende Verletzungen

2. Explosionsverletzung

3. Schuss- oder Stichverletzung

4. Schwerste multiple stumpfe Verletzungen durch Überroll- oder Anpralltrauma

5. Verbrennung

6. Verletzungen durch CBRN-Agenzien

Aufgrund der zu erwartenden Verletzungsmuster ist auf Bevorratung von Material zur Versorgung penetrierender Verletzungen in ausreichender Anzahl und schneller Verfügbarkeit $\mathrm{zu}$ achten, Außerdem kommt das C-ABCDESchema zur Anwendung - mit dem Ziel, lebensbedrohliche Blutungen an den Extremitäten schnellstmöglich $\mathrm{zu}$ stoppen.

\section{Transport}

Der schnellstmögliche Start der Transportkette in Richtung sicherer Bereiche ist ein wesentlicher Schritt zur Rettung von Opfern. Für den Transport der Schwerverletzten müssen Transporthilfen (z.B. Bergetücher, Spineboards) bereitgestellt werden. Es wird nochmals betont, dass der Start der gerichteten Transportkette im unsicheren Bereich durch die die Polizei erfolgen muss.

Auf Seiten des Rettungsdienstes ist die möglichst frühzeitige Bereitstellung von Transportkapazitäten ein wichtiger Punkt. Hierzu ist die Kenntnis der zu erwartenden Zahl an Betroffenen und davon potenziell lebensbedrohlich Verletzten essenziell (s. Ersteinschätzung).

Da mit einer schnellen Evakuierung und einem schnellen Abtransport der rot gesichteten Patienten $\mathrm{zu}$ rechnen ist (hier v.a. penetrierende Traumata und schwerste stumpfe Verletzungen nach
Überroll- oder Anpralltrauma), kommt den aufnehmenden Krankenhäusern eine besondere Bedeutung zu. Die Vorbereitungszeit kann extrem kurz sein, die Anzahl der aufzunehmenden Opfer wird sich ggf. von den prinzipiell getroffenen Vereinbarungen deutlich unterscheiden und die Patienten werden medizinisch nur mit dem Nötigsten versorgt sein. Daher ist eine frühestmögliche Information der Krankenhäuser über die aktuelle Lage und die voraussichtliche Patientenzahl einschließlich der Verletzungsschwere wichtig, um eine adäquate Vorbereitung der Krankenhäuser zu ermöglichen. Die Kommunikationswege und Informationsverfahren sollten im Vorfeld festgelegt und regelmäßig geübt werden.

\section{Besondere Patientengruppen}

Die Versorgung von Kindern im Rahmen eines terroristischen Anschlages stellt eine besondere Herausforderung dar. Dies betrifft sowohl die fachliche Qualifikation als auch die materiellen Ressourcen, die zur Versorgung von Kindern benötigt werden. Die Erarbeitung von Empfehlungen zu dieser Patientengruppe war im Rahmen der Ergebniskonferenz aus zeitlichen Gründen nicht umsetzbar und wird zu einem späteren Zeitpunkt erfolgen. Grundsätzlich gilt, dass bestehende MANV-Konzepte die Behandlung von Kindern berücksichtigen sollten und diese Konzepte auf die Spezifika bei lebensbedrohlichen Einsatzlagen adaptiert werden müssen. Gleiches gilt für die Behandlung von Verbrennungspatienten.

Auch auf die Versorgung von weiteren Patientengruppen, wie eine größere Anzahl von fremdsprachigen Patienten oder von Patienten mit schlechterem Allgemeinzustand, z. B. bei an Anschlägen auf medizinische Versorgungseinrichtungen, konnte in dem zitierten Rahmen nicht mehr eingegangen werden.

\section{Umgang mit unverletzt Betroffenen}

Im Rahmen einer lebensbedrohlichen Einsatzlage ist mit einer großen Anzahl unverletzter Betroffener zu rechnen. Für diese muss eine Betreuung eingerichtet werden.

Für die Polizei spielen alle Personen an der Schadensstelle als potenzielle Zeugen im Rahmen der Ermittlungsarbeit eine bedeutende Rolle. Durch die Polizei erfolgt die sog. taktische Betreuung, um die relevanten Zeugen herauszufiltern. Hier soll eine enge Abstimmung mit der psychosozialen Notfallversorgung stattfinden.

\section{Erfassung von Patientendaten}

Die Identifizierung von Opfern und Betroffenen spielt eine herausragende Rolle bei der Bewältigung lebensbedrohlicher Einsatzlagen. Zur Registrierung sollen die üblichen Dokumentationssysteme verwendet werden:

- Patientenanhängekarte,

- Einsatzprotokolle,

- vorhandene Dokumentations- und

Registrierungswerkzeuge für den

MANV.

Zur möglichst vollständigen Registrierung ist eine enge Zusammenarbeit zwischen polizeilicher und nichtpolizeilicher Gefahrenabwehr erforderlich. Die zuständigen Behörden sollten den Prozess der Registrierung zwischen Präklinik und Klinik abstimmen und synchronisieren. Es ist davon auszugehen, dass die Registrierung am Schadensort mit erheblichen Schwierigkeiten verbunden sein wird und eine sichere und endgültige Dokumentation und Identifizierung erst in den Krankenhäusern erfolgen kann. Umso wichtiger ist die enge Verknüpfung zwischen der präklinischen und klinischen Identifizierung. Im Idealfall wird hier ein einheitliches System verwendet.

Vorhandene elektronische Auskunftssysteme wie z.B. gsl.net müssen unterhalb der Katastrophenschwelle nutzbar sein; ggf. müssen Rechtsgrundlagen angepasst werden. Bei gsl.net handelt es sich um ein einheitliches elektronisches Dokumentationssystem der Polizei. Dieses ist darauf ausgelegt, bei Großschadenslagen eine einheitliche und transparente Erfassung der Personendaten von Betroffenen und Verletzten sicherzustellen. 


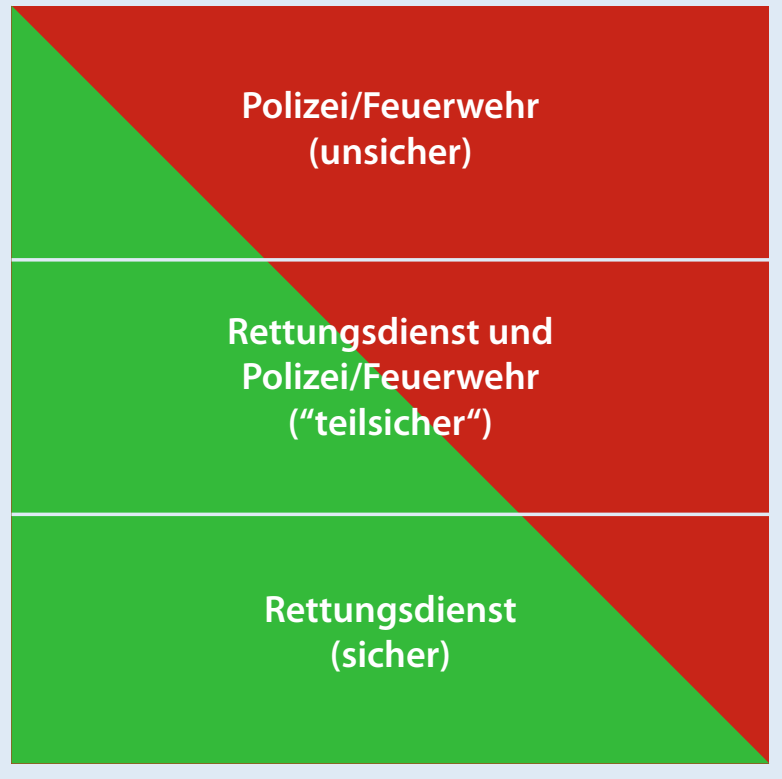

Die Einrichtung einer zentralen Personenauskunftsstelle ist notwendig. Die Verfahren müssen etabliert sein und regelmäßig geübt werden.

\section{Diskussion und zusammen- fassende Bewertung}

Die hier dargestellten Ergebnisse sind das Resultat eines strukturierten nationalen Auswerteprozesses. Sie stammen von Experten der an der Bewältigung lebensbedrohlicher Einsatzlagen beteiligten Behörden, Institutionen und Organisationen. Damit stellen sie eine Expertenmeinung dar, die auf der Basis aller bisher vorliegenden Erkenntnisse gefasst wurde. In Deutschland vorhandene Konzepte wurden, soweit sie vorlagen, ausgewertet und z. T. als Basis in die vorliegenden Resultate integriert.

In allen Arbeitssitzungen und Diskussionen herrschte Einigkeit darüber, dass das Vorhandensein eines allgemeinen MANV-Konzepts sowie eine gute Schulung und Übung dieses Konzepts eine unverzichtbare Basis für die Vorbereitung auf lebensbedrohliche Einsatzlagen darstellt. Erst aufbauend auf diese Basis können die Spezifika erarbeitet und eingeplant, geschult und geübt werden.

Weiterhin bestand Übereinkunft darüber, dass die jeweiligen Spezifika lebensbedrohlicher Einsatzlagen und die
Konsequenzen für den Einsatz in möglichst einfachen Handlungsempfehlungen dargestellt, geschult und geübt werden müssen.

Ein Kernstück der Zusammenarbeit zwischen der polizeilichen und nichtpolizeilichen Gefahrenabwehr ist neben einer gut abgestimmten Kommunikation auf allen Ebenen die Rettung von Betroffenen und damit die Etablierung der gerichteten Transportkette mit den jeweiligen Knotenpunkten. In diesem Zusammenhang lassen sich beispielsweise die Aufgaben der Polizeikräfte in wenigen Punkten zusammenfassen. Sobald die genuinen Aufgaben der Polizei es zulassen, sind

- Ersteinschätzung,

- Blutstillung und

- Start der gerichteten Transportkette in Richtung sicherer Bereiche

wichtige und prioritäre Punkte im Sinne der Rettung von Betroffenen.

Für den Rettungsdienst lassen sich folgende vorrangigen Schritte herausarbeiten:

- Eigensicherung,

- Start der Vorsichtung,

- „Finde die Roten“,

- Durchführung lebensrettender Sofortmaßnahmen,

- schnellstmöglicher Transport der Patienten der Sichtungskategorie I
Rot ins Krankenhaus. (Gerade bei penetrierenden Verletzungen am Rumpf ist davon auszugehen, dass eine lebensrettende Versorgung nur innerklinisch durch eine chirurgische Intervention erfolgen kann. Daher ist dieses Vorgehen eine wesentliche Besonderheit, die es zu beachten gilt.)

Die hier vorgestellten Resultate zum Thema Sichtung bauen auf die Ergebnisse der 7. Sichtungs-Konsensus-Konferenz unter Federführung des BBK auf [12]. Hier wurde Sichtung als Prozess beschrieben und konsentiert. An erster Stelle steht die Ersteinschätzung (•Abb. 1). Diese hat eine erste grobe Abschätzung der Anzahl der Opfer zum Ziel, ebenso soll eine ungefähre Angabe der potenziell lebensbedrohlich verletzten Patienten gegeben werden. Da ausschließlich die Polizei bei lebensbedrohlichen Einsatzlagen im unsicheren Bereich agiert, wird die Aufgabe der Ersteinschätzung auch der Polizei zukommen (-Abb. 2). Die Brisanz der Ersteinschätzung muss sowohl den Polizeikräften als auch dem Rettungsdienst und dem Personal der Leitstellen bekannt gemacht, geschult und geübt werden.

》) Bei der Raumordnung muss ein Bereich definiert werden, in dem der Rettungsdienst tätig sein kann

An die Ersteinschätzung schließt sich die Vorsichtung an. Diese wird durch das Personal des Rettungsdienstes vorgenommen und sollte nur in weitestgehend gesicherten Bereichen stattfinden (teilsicher). Ob die Vorsichtung an geschützten Patientenablagen oder beispielsweise in gesicherten Korridoren abläuft, ist lageabhängig und kann hier nicht festgelegt werden.

Intensiv diskutiert wurde die Aufteilung der Raumordnung. Insgesamt wurde die Dreiteilung in unsicher, teilsicher und sicher als kritisch angesehen. Gerade die Festlegung des teilsicheren Bereichs könnte im Einsatz mit Schwierigkeiten und Unsicherheiten verbunden sein. Besonders erwähnenswert ist hier die Schwierigkeit aufder Basis eines meist 


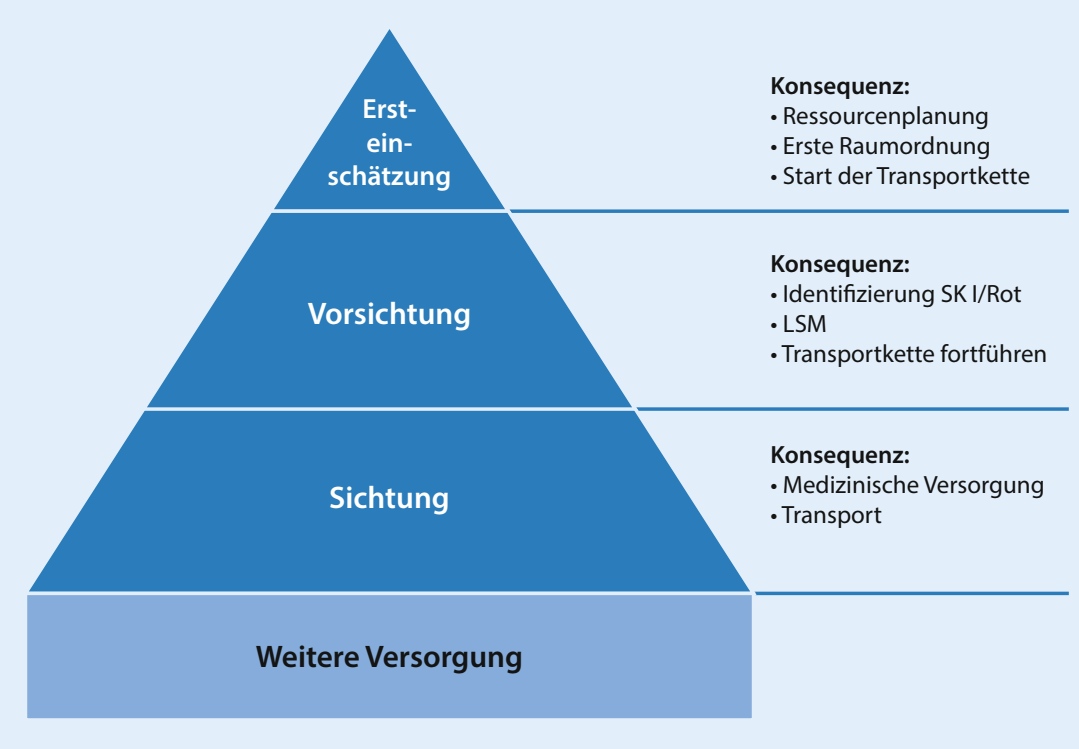

Abb. 2 \ Sichtungsprozess

noch nicht vollständig geklärten Lagebilds eine solche Festlegung zu treffen. Insbesondere betrifft dies den teilsicheren Bereich. Es bleiben hier eine Restunsicherheit und ein Restrisiko bestehen. Auf der anderen Seite würde ein Verzicht auf die Festlegung des teilsicheren Bereichs den unsicheren Bereich derart vergrößern, dass der Rettungsdienst und damit die medizinische Versorgung erst in erheblichem räumlichem Abstand (im sicheren Bereich) erfolgen würde, was $\mathrm{zu}$ einer Verzögerung und damit Verschlechterung der Patientenversorgung führen würde. Im Rahmen der Kontroverse bestand aber Einigkeit darüber, dass festgelegt werden muss, in welchem Bereich Kräfte der nichtpolizeilichen Gefahrenabwehr mit vertretbarem Risiko agieren können. Ebenso muss klar sein, welche Maßnahmen in diesen Bereichen stattfinden dürfen und dass die Verweildauer in diesen Bereichen so kurz wie möglich zu halten ist. Die Aufteilung und Benennung in unsicher, teilsicher und sicher war die von der Konferenz präferierte Nomenklatur. Entscheidend ist aber die klare Absprache zwischen polizeilichen und nichtpolizeilichen Kräften, die lagebezogene Festlegung dieser Bereiche und die Vermittlung von Handlungssicherheit, wie in diesen Bereichen vorzugehen ist. Die diagonale Teilung der Bereiche in der $\bullet$ Abb. 1 und der Anteil der Farben rot und grün in den jeweiligen Bereichen stellt diese Restunsicherheit dar.

\section{Besondere Vorsichtsmaßnahmen}

Aus Gründen des Eigenschutzes soll mitgeführtes Gepäck von Betroffenen/ Verletzten an der Einsatzstelle verbleiben und darf keinesfalls mit in die Rettungswagen/Transportfahrzeuge genommen werden. $\mathrm{Zu}$ diesem Zweck sollten Sammelstellen für Gepäck eingerichtet werden, die sich räumlich deutlich abgesetzt von etwaigen Patientenablagen/Ladezonen befinden sollten.

Obwohl die Polizei im unsicheren Bereich versuchen wird, mitgeführte Waffen oder Sprengstoffvorrichtungen bei potenziellen Tätern zu detektieren und darauf entsprechend mit polizeilichen Maßnahmen zu reagieren, ist es dennoch möglich, dass bewaffnete Personen mit dem Rettungsdienst in Kontakt kommen. Die nichtpolizeiliche Gefahrenabwehr sollte daher bei der Vorsichtung der Patienten im Rahmen des Bodychecks darauf achten, ob sich möglicherweise Waffen (z. B. Schusswaffen oder Messer) und/oder Sprengstoffwesten/-gürtel an den Patienten befinden. Bei Feststellung derartiger Gegenstände sind umstehende Kräfte sofort per Ausruf zu warnen. Darüber hinaus ist sofort die Polizei hin- zuzuziehen. Diese wird dann die Waffe sicherstellen/beschlagnahmen und erforderlichenfalls über die Hinzuziehung von Spezialkräften (z.B. Entschärfer) entscheiden. Bei der Feststellung von sprengstoffverdächtigen Gegenständen sind keinesfalls eigene Entschärfungsversuche durchzuführen. Stellt die Polizei eine entsprechende Gefährdungslage fest, sind so schnell wie möglich alle Verletzten/Betroffenen/Einsatzkräfte aus dem möglichen Gefahrenbereich zu evakuieren.

Wird einem Patienten im Rahmen der Behandlung durch Kräfte der nichtpolizeilichen Gefahrenabwehr eine Waffe abgenommen, sollte diese unverzüglich und so an die Polizei übergeben werden, dass die Polizeikräfte aus der Situation keinesfalls eine Eigengefährdung ableiten können.

\section{Einsatzauswertung und Qualitätsindikatoren}

Für eine systematische Darstellung und Evaluation von rettungsdienstlichen Einsätzen bei lebensbedrohlichen Einsatzlagen existiert derzeit noch keine einheitliche Grundlage. Nach den Attentaten von Paris gab es Veröffentlichungen in renommierten internationalen Journals, welche die Ereignisse dieser Nacht vom 13.11.2015 schildern [13-15]. Allerdings sind die Berichte weniger als systematische Einsatzdokumentation und -auswertung denn als kostbare Erfahrungsberichte zu lesen. Eine Veröffentlichung des Französischen Gesundheitsministeriums zur Pariser Terrornacht beschreibt eindrücklich die „lessons learned“ [15]. Die neueste Veröffentlichung fasst die konkreten Konsequenzen und deren Umsetzung hervorragend zusammen [16].

Will man strukturiert die Lehren aus derartigen Einsätzen ziehen, so besteht ein dringender Bedarf nach einer systematischen Auswertung anhand fester Kriterien [17].

Kürzlich wurde ein Modell mit dem Ziel veröffentlicht, Qualitätsindikatoren und Einsatzmerkmale für lebensbedrohliche Einsätze wie Terror oder Amok zu definieren. Anhand der hier definierten Parameter können lebensbedrohli- 
che Einsatzlagen systematisch evaluiert und damit letztlich vergleichbar gemacht werden [18]. Nachdem der Würzburger Terroranschlag vollständig nach diesem Schema ausgewertet wurde, muss sich dieses System nun bei der Auswertung andere Anschläge bewähren.

\section{Fazit für die Praxis}

- Der Schwerpunkt in dieser Veröffentlichung liegt auf dem präklinischen Management. Das Vorhandensein gut funktionierender und geübter MANV-Konzepte ist eine wichtige Voraussetzung für die erfolgreiche Planung und Bewältigung lebensbedrohlicher Einsatzlagen. Auf diese Konzepte aufbauend müssen die wesentlichen Spezifika erkannt und eingearbeitet werden.

- Eine obligatorische Grundlage für die erfolgreiche Einsatzbewältigung ist die enge Zusammenarbeit zwischen polizeilicher und nichtpolizeilicher Gefahrenabwehr. Diese Zusammenarbeit kann nicht erst im Einsatz beginnen, sondern muss bereits im Vorfeld bei der Benennung gemeinsamer Ziele und Strategien und gemeinsamen Übungen gelebt werden.

- Die hier vorgestellten Ergebnisse sind als Handlungsempfehlungen zu verstehen, die von Experten erstellt wurden und all denjenigen eine Orientierung geben sollen, die sich mit der Bewältigung lebensbedrohlicher Einsatzlagen befassen.

\section{Korrespondenzadresse}

\section{T. Wurmb}

Sektion Notfall- und Katstrophenmedizin, Klinik und Poliklinik für Anästhesiologie, Universitätsklinikum Würzburg Oberdürrbacher Straße 6, 97080 Würzburg, Deutschland wurmb_t@ukw.de

\section{Einhaltung ethischer Richtlinien}

Interessenkonflikt. T. Wurmb, B. Kowalzik, J. Rebuck, A. Franke, D. Cwojdzinski, N. Bernstein, T. Brodala und M. Weber geben an, dass kein Interessenkonflikt besteht.
Dieser Beitrag beinhaltet keine von den Autoren durchgeführten Studien an Menschen oder Tieren.

Open Access. Dieser Artikel wird unter der Creative Commons Namensnennung 4.0 International Lizenz (http://creativecommons.org/licenses/by/4.0/deed. de) veröffentlicht, welche die Nutzung, Vervielfältigung, Bearbeitung, Verbreitung und Wiedergabe in jeglichem Medium und Format erlaubt, sofern Sie den/die ursprünglichen Autor(en) und die Quelle ordnungsgemäßnennen, einen Linkzur Creative Commons Lizenz beifügen und angeben, ob Änderungen vorgenommen wurden.

\section{Literatur}

1. Wurmb T, Hossfeld B, Zoller G (2018) Polizei und Rettungsdienst bei der Bewältigung lebensbedrohlicher Einsatzlagen - Unterschiedliche Strukturen, Gemeinsames Vorgehen. Notfallund Rettungsmedizin. https://doi.org/10.1007/ s10049-018-0454-3

2. Autrey AW, Hick JL, Bramer K et al (2014) 3 Echo: concept of operation sfor early care and evacuation of victims of mass violence. Prehosp Disaster Med 29:421-428

3. Jacobs LM, Wade DS, McSwain A et al (2013) The Hartford Consensus: THREAT, a medical disaster preparedness concept.JAm Coll Surg 217:947-953

4. Wurmb T, Helm M, Hossfeld B (2018) Präklinische Versorgung bei Terror- und Amoklagen. In: Deutsche Akademie für Anästhesiologische Fortbildung (Hrsg) Refrescher Course - Aktuelles Wissen für Anästhesisten. Nr. 44. Aktiv Druck, Nürnberg, S277. ISBN 978-3932653537

5. Hossfeld B, Adams HA, Bohnen R et al (2017) Zusammenarbeit von Rettungskräften und Sicherheitsbehörden bei bedrohlichen Lagen. Anästh Intensivmed 58:573-583

6. Friemert B, Franke A, Bieler D, Achatz A, Hinck D, Engelhardt $M$ (2017) Versorgungsstrategien beim MANV/TerrorMANV in der Unfall- und Gefäßchirurgie - Darstellung eines Versorgungskonzeptes. Chirurg 88:856-862

7. Güsgen C, Franke A, Hentsch S, Kollig E, Schwab $R$ (2017) Das Terroranschlagtrauma - eine eigene Entität des Polytraumas. Chirurg 88:821-829

8. Wurmb T, Scholtes K, Kolibay F (2017) Die Rolle der Krankenhäuser bei der Bewältigung von Bedrohungslagen. BBK Bevölkerungsschutz 2:28-29

9. Friemert B, Franke A, Bieler D et al (2017) Versorgungsstrategien beim MANV/Terror-MANV in der Unfall- und Gefäßchirurgie. Chirurg 88:856-862

10. Franke A, Bieler B, Friemert B et al (2017) Präund Innerklinisches Management bei MANV und Terroranschlag. Chirurg 88:830-840

11. Heller AR, Salvador N, Frank M, Schiffner J, Kipke R, Kleber C (2017) Diagnostische Güte von Vorsichtungsalgorithmen für den Massenanfall von Verletzten. Anaesthesist 66:762-772

12. Bundesamt für Bevölkerungsschutz und Katastrophenhilfe (2017) 7. Sichtungs-Konsensus-Konferenz. https://www.bbk.bund.de/ SharedDocs/Downloads/BBK/DE/Downloads/ GesBevS/7_Sichtungs-Konsensus-Konferenz. pdf?_blob=publicationFile. Zugegriffen: 10.07.2018

13. Hirsch M, Carli P, Nizard R, health professionals of Assistance Publique-Hôpitaux de Paris (APHP) et al (2015) The medical response to multisite terrorist attacks in Paris. Lancet 386:2535-2538
14. Haug CJ (2015) Report from Paris. N Engl J Med 373(27):2589-2593

15. Philippe JM, Brahic O, Carli P et al (2016) French Ministry of Health's response to Paris attacks of 13 November 2015. Crit Care 20:85-86

16. Carli P, Pons F, Levraut J et al (2017) The French emergency medical services after the Paris and Nice terrorist attacks: what have we learnt? Lancet 390(10113):2735-2738. https://doi.org/10.1016/ S0140-6736(17)31590-8

17. Goralnick E, Van Trimpont F, Carli P (2017) Preparing the next terrorism attack: lessons from Paris, Brussels and Boston. JAMA Surg 152:419-420. https://doi.org/10.1001/jamasurg. 2016.4990

18. Wurmb T, Justice P, Dietz S et al (2017) Mögliche Qualitätsindikatoren und Einsatzmerkmale für rettungsdienstliche Einsätze bei Terroranschlägen oder anderen Bedrohungslagen - Eine Pilotstudie nach dem Würzburger Terroranschlag vom Juli 2016. Anaesthesist 66:404-411 